\title{
Hot balloon ablation of persistent atrial fibrillation with a dilated left atrium in a case of dilated cardiomyopathy
}

Ayako Okada*, Morio Shoda, Takahiro Takeuchi, Ken Kato, Wataru Shoin, Takahiro Okano, Hideki Kobayashi, Yasutaka Oguchi, Koichiro Kuwahara

Department of Cardiovascular Medicine, Shinshu University School of Medicine, Japan

Received: January 2, 2017

DOI: $10.5430 /$ ijdi.v4n2p28
Accepted: March 30, 2017

Online Published: April 5, 2017

\begin{abstract}
A 70-year-old man who developed dilated cardiomyopathy with persistent atrial fibrillation (PSAF) was admitted to our hospital. He was already drug-resistant. The left atrium (LA) was severely enlarged. The left ventricular ejection fraction (LVEF) was 39\%, and contraction was severely reduced. For AF ablation, catheter ablation (CA) was used for mild to moderate LV dysfunction without severe left atrial dilatation. In severe LV dysfunction, excessive intravenous drip volume may precipitate congestive heart failure. On the other hand, shorter isolation time has been reported with balloon ablation as compared to CA. Therefore, the intravenous drip volume is small. We planned to use the balloon technique for a patient with LA dilatation and severe LV dysfunction despite another hospital not having done so. After the procedure, the pulmonary veins were successfully isolated, and congestive heart failure recovered after sinus rhythm termination. There was no recurrence of AF after the procedure. Hot balloon ablation can be effective for PSAF with severe heart failure.
\end{abstract}

Key Words: Hot balloon, Dilated cardiomyopathy, Left ventricular dysfunction, Persistent atrial fibrillation, Pulmonary hypertension

\section{INTRODUCTION}

Atrial fibrillation (AF) is the most common chronic arrhythmia. For AF ablation, catheter ablation (CA) is used for mild to moderate left ventricular dysfunction without severe left atrial (LA) dilatation. ${ }^{[1]}$ The reason for this is that congestive heart failure occurs after ablation because of the intravenous drip volume with severe left ventricular (LV) dysfunction. On the other hand, it has been reported that the time of isolation can be shorter with balloon ablation than with $\mathrm{CA}_{;} ;^{[1,2]}$ therefore, the intravenous drip volume is small. ${ }^{[3-5]}$ We decided to use the balloon technique for a patient with LA dilatation and severe LV dysfunction.

\section{CASE REPORT}

The patient was a 70-year-old man who developed dilated cardiomyopathy with congestive heart failure and was admitted to our hospital. He had also developed persistent AF (PSAF), but there was no indication for CA in the previous hospitalization.

His congestive heart failure did not recover because of $\mathrm{AF}$, though he was taking amiodarone $(200 \mathrm{mg} / \mathrm{day})$ and carvedilol $(6.25 \mathrm{mg})$. He was already drug-resistant.

\footnotetext{
*Correspondence: Ayako Okada; Email: ayakookada1026@yahoo.co.jp; Address: Department of Cardiovascular Medicine, Shinshu University School of Medicine, 3-1-1 Asahi, Matsumoto, Nagano 390-8621, Japan.
} 
Echocardiography was performed at admission. The LA diameter (LAD) was $5.67 \mathrm{~cm}$, and the LA volume index (LAVI) was $72.9 \mathrm{ml} / \mathrm{m}^{2}$. The LV ejection fraction was $39 \%$ (modified Simpson method), and LV contraction showed diffuse severe hypokinesis.

Treatment with AF ablation with the balloon technique was chosen. However, the pulmonary vein (PV) was large, especially the left superior pulmonary vein (LSPV) (see Figure 1). Since the cryoballoon diameter was $28 \mathrm{~mm}$, the cryoballoon would not occlude the LSPV with a long axis dimension $>29 \mathrm{~mm}$.

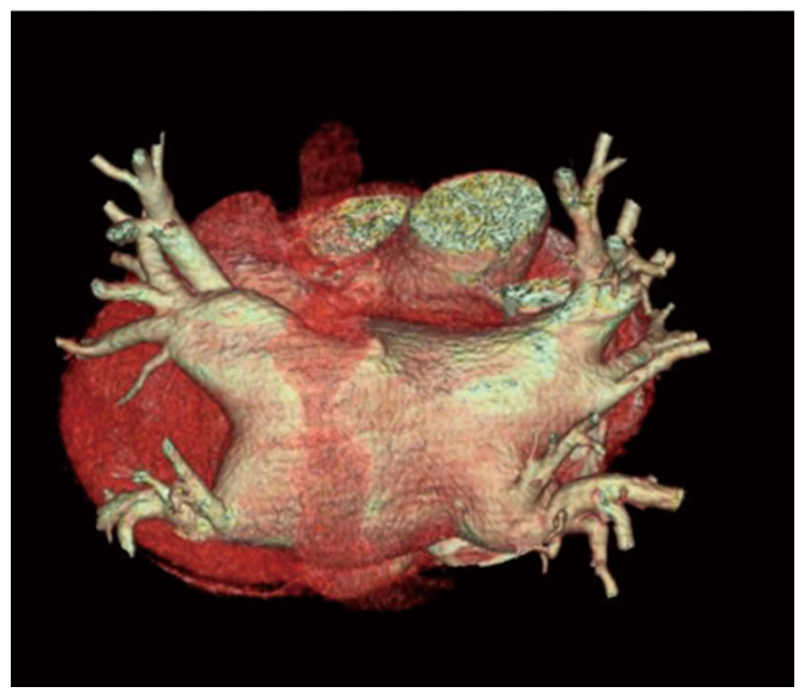

Figure 1. CT findings of the left atrium and the 4 pulmonary veins

The balloon size of the SATAKE Hot balloon (TORAY, Tokyo Japan) can be changed by an inflatable balloon with saline and contrast (Iopamiron, Bayer Global Pharmaceuticals Consumer Health, Bayer, Tokyo, Japan). The ablation procedure was performed under general anesthesia with Precedex (Maruishi Pharmaceutical, Osaka Japan), but no intubation was needed. Esophageal temperature was monitored using a gastric tube. Transseptal access was achieved using a radiofrequency (RF) needle (Japan Lifeline Co., Ltd, Tokyo, Japan) and an 8.5 Fr SL0 sheath (St. Jude Medical, Inc, St Paul, MN, USA).

A 3D-electroanatomical mapping system (CARO, Biosense Webster, Diamond Bar, CA, USA Inc), was used. Four polar superior vena cava (SVC) and 10 polar coronary sinus electrodes were positioned through the right jugular vein (BeeAT, Japan Lifeline Co., Ltd). Ten polar electrodes were positioned in the SVC for phrenic nerve stimulation and the right ventricular apex for bradycardia pacing.

During the procedure, the temperature of the central balloon Published by Sciedu Press was set at $70^{\circ} \mathrm{C}$. After LA septal puncture, the guidewire was placed into the right superior pulmonary vein (RSPV), dragging the balloon into the RSPV ostium. The balloon was positioned under a biplane fluoroscopic view. The balloon was inflated over the PV size because of PV stenosis. The inflated balloon was seen to occlude the RSPV on venography. There was no leakage into the LA after injection or after contrast medium injection. The balloon central temperature achieved $70^{\circ} \mathrm{C}$, with ablation for 2 minutes. The amount of contrast medium in the balloon was $12 \mathrm{ml}$. There were small indentations at the top and bottom of the balloon. Next, the balloon was positioned in the RSPV antrum.

The balloon was fully expanded without indentation with $15 \mathrm{ml}$ of contrast medium, with ablation for 2 minutes 30 seconds. Then, $5 \mathrm{ml}$ of contrast medium in the balloon were removed, and the balloon was in contact with the RSPV carina. The procedure time at the RSPV carina was 2 minutes 30 seconds. The balloon was positioned at the right inferior pulmonary vein (RIPV) ostium with $15 \mathrm{ml}$ of contrast medium. There was no leakage into the LA antrum after injection of the contrast medium. The balloon ablation lasted 1 minute 50 seconds. The balloon size was increased by adding $3 \mathrm{ml}$ of contrast medium. The RIPV antrum was ablated for 2 minutes 30 seconds with a fully expanded balloon.

The balloon was positioned in the LSPV. The inflated balloon was wedged at the LSPV, and there were indentations at the top and bottom of the balloon.

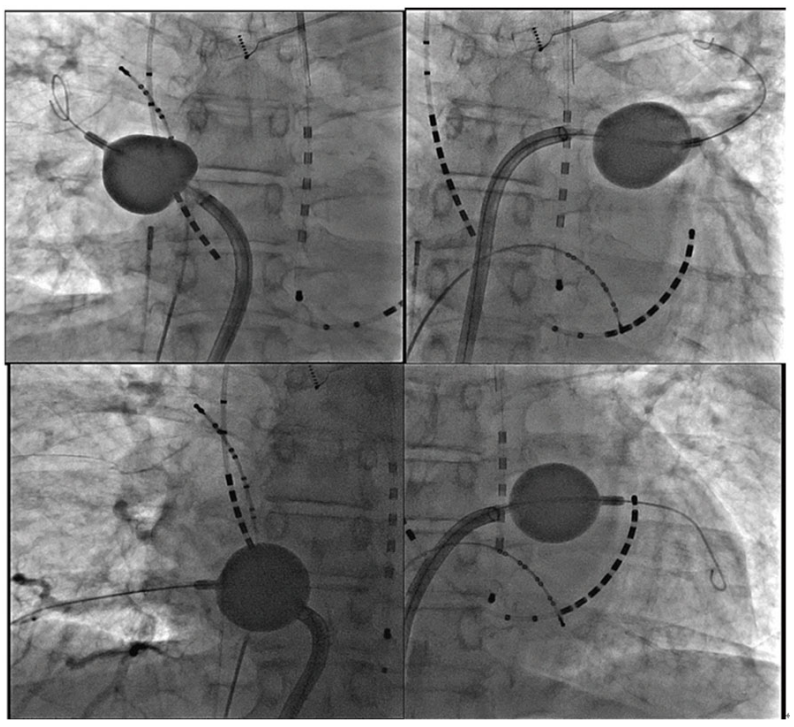

Figure 2. Pulmonary veins are occluded by a hot balloon catheter at the ostium

There was no leakage of contrast medium after venography. The balloon ablated the LSPV ostium for 3 minutes $30 \mathrm{sec}$ onds. Then, $2 \mathrm{ml}$ of contrast medium were removed from 
the balloon, and the balloon was in contact with the LSPV antrum, which was ablated for 3 minutes 30 seconds. Finally, the balloon was positioned in the left inferior pulmonary vein (LIPV). The inflated balloon needed only $9 \mathrm{ml}$ of contrast medium, and ablation lasted 3 minutes 30 seconds (see Figure 2).
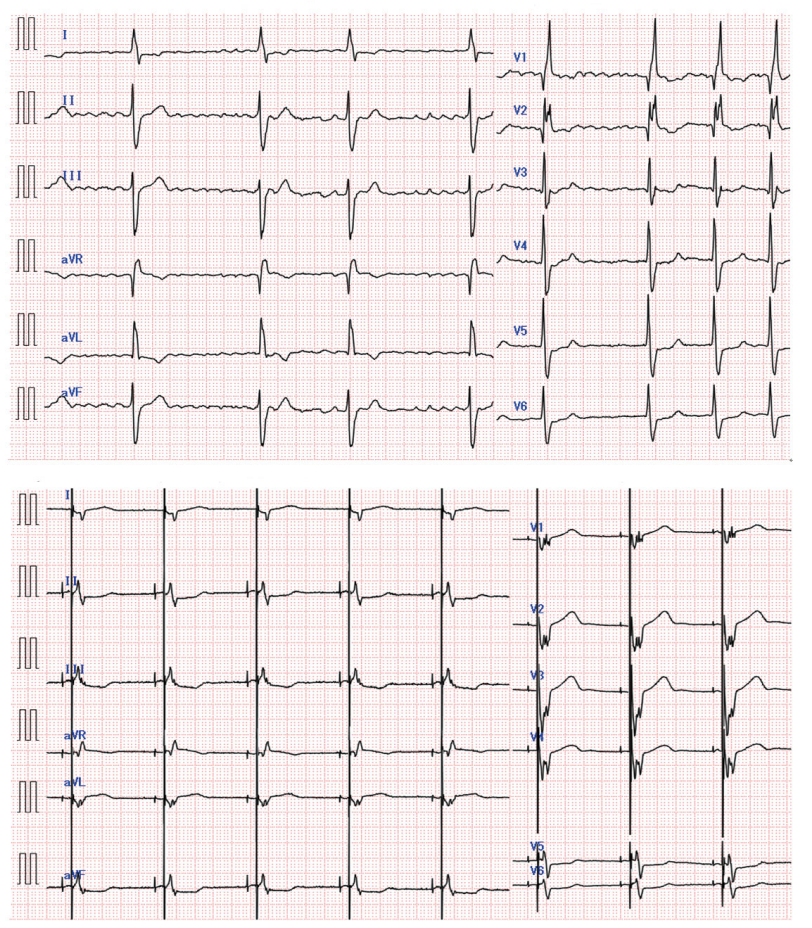

Figure 3. Pre ablation (superior) and post ablation (posterior)

During LPV ablation, the esophageal temperature was higher than $41^{\circ} \mathrm{C}$. Cooling saline and low contrast medium were put into the gastric tube.

After the procedures, the balloon catheter was removed, and the Lasso catheter (Biosense Webster, Inc) was taken into the 4 PVs.

There were no PV potentials in each of the 4 PVs during the LA potential. There were no complications during the procedure.

The patient also developed atrial flutter. Therefore, cavotricuspid isthmus ablation was needed for atrial flutter. A tricuspid isthmus block line was achieved successfully.

After ablation therapy, the patient was implanted with a defibrillator with cardiac resynchronized therapy because of a low ejection fraction and bradycardia due to atrioventricular block (see Figure 3).

There was no recurrence of AF in this patient 6 months after the procedure.

\section{Discussion}

With respect to long-term outcomes following hot balloon ablation, the AF-free rate has been reported to be $64.7 \%$. ${ }^{[3]}$ The independent predictor of AF recurrence was heart failure with preserved ejection fraction $(\mathrm{EF}) .{ }^{[4,5]}$ In a previous study, ${ }^{[4]} 92$ patients were free from AF (60 paroxysmal, 32 persistent), but cases with severe heart failure and a dilated left atrium ( $>55 \mathrm{~mm}$ ) were excluded. However, in the present case, the patient developed decompensated heart failure with severe LV dysfunction, and hot balloon ablation was effective for congestive heart failure with a giant left atrium.

\section{Conclusion}

Successful PV isolation can be performed in a patient with dilated cardiomyopathy using hot balloon ablation.

\section{CONFlicts of INTEREST Disclosure}

The authors have declared no conflicts of interest.

\section{REFERENCES}

[1] Kuhne M, Suter Y, Altmann D, et al. Cryoballoon versus radiofrequency catheter ablation of paroxysmal atrial fibrillation: Biomarkers of myocardial injury, recurrence rates, and pulmonary vein reconnection patterns. Heart Rhythm. 2010; 7: 1770-1776. PMid:20817019 https://doi.org/10.1016/j.hrthm.2010.08.028

[2] Schmidt M, Dorwarth U, Anderesen D, et al. German ablation reg istry: Cryoballoon vs radiofrequency ablation in paroxysmal atrial fibrillation-one-year outcome data. Heart Rhythm. 2016; 13: 836-844. PMid:26681608 https ://doi.org/10.1016/j.hrthm.2015.1 2.007
[3] Yamaguchi Y, Sohara H, Takeda H, et al. Long-term results of radiofrequency hot balloon ablation in patients with paroxysmal atrial fibrillation. J Cardiovasc Electrophysiol. 2015; 26: 1298-1306 PMid:26331460 https ://doi.org/10.1111/jce.12820

[4] Sohara H, Takeda H, Ueno H, et al. Feasibility of the radiofrequency hot balloon catheter for isolation of the posterior left atrium and pulmonary veins for the treatment of atrial fibrillation. Circulation: Arrhythmia and Electrophysiology. 2009; 2: 225-232. https://doi.org/10.1161/circep.108.817205

[5] Metzner A, Wissner E, Lin T, et al. Balloon devices for atrial fibrillation therapy. Circulation: Arrhythmia and Electrophysiology. 2015; 4: 58-61. https://doi.org/10.15420/aer.2015.4.1.58 